will provide both a comprehensive source of information and a stimulus for researchers in fungal biology.

JEFF BOND

Institute of Animal Genetics University of Edinburgh

Banbury Report 25: Nongenotoxic Mechanisms in Carcinogenesis. Edited by BYRON E. BUTTERWORTH and Thomas J. Slaga. New York: Cold Spring Harbor Laboratory. 1987. 397 pages. Cloth \$70.00. ISBN 0879692251.

Coincident with the encroaching confusion in genetic toxicology over the role it has to play in carcinogen prediction (Tennant et al. (1987), Science 236, 933941), there has been a resurgence of interest in nongenotoxic factors in carcinogenesis. This interest is encouraged by this book which, wisely, does not narrow its field of view exclusively to so-called nongenotoxic carcinogens. Chemicals thought to be nongenotoxic to-day may very well be demonstrated to have genotoxic potential in the future. Nevertheless, the re-designation of a chemical as genotoxic does not necessarily mean that its carcinogenic activity is through a genotoxic mechanism. For example, trichloroethylene may be a mouse hepatocarcinogen because of its effect on peroxisomes - it is nongenotoxic in a battery of in vitro assays - but its metabolites (e.g. chloral) can certainly induce mutation, the absence of a significant response in the in vitro assays being a result of the inadequate concentrations of the mutagenic metabolites achieved in those tests. But the mere demonstration of a genotoxic metabolite from trichloroethylene does not imply that it is, after all, a genotoxic carcinogen: the dominant effect may still be peroxisomal proliferation, followed by oxidative initiation and/or promotion, or other process. Conversely, dichloromethane is strongly genotoxic, in bacterial assays, but recent work has shown that the bacterial metabolism of this compound is quite different from its mammalian metabolism and that it does not interact covalently with DNA in rats (Green et al. (1988), Toxicol. Appl. Pharmacol. 93, 1-10).

It is not a corollary of nongenotoxic carcinogenesis that the task of anyone concerned with regulation is made easier. The theoretical expectation of a threshold effect is difficult to realise. Thus, amitrole induces rat thyroid tumours at $100 \mathrm{ppm}$ in the diet, but not at 10 or $1 \mathrm{ppm}$ (Steinhoff et al. (1983), Toxicol. Appl. Pharmacol. 69, 161-169). Unfortunately, it is very rare for an experimental design to permit risk assessments to be made; experiments are almost always designed only to identify hazard. Consequently, it is difficult to conclude that $10 \mathrm{ppm}$ amitrole is below the thyroid carcinogenic threshold.

The multi-step process of carcinogenesis is influenced by a large number of factors, some of which give the appearance of being at least as important as genotoxicity. This is not a new idea, but the real value of this book lies in its summary of some of these processes, review of a number of the chemical carcinogens which are nongenotoxic and - most important - stimulation of genotoxicity researchers to consider more seriously in vivo responses other than mutation. Dietary factors, such as deficiencies in the lipotropes choline and methionine or over-feeding, can significantly alter tumour incidence. This issue receives well-deserved attention in this book. The role of chronic hyperplasia is addressed in discussion of the hepatocarcinogenesis of many pesticides and $\operatorname{di}(2-$ ethylhexyl)phthalate (DEHP), the urinary tract carcinogenesis of nitrilotriacetate and a number of substances which can lead to calculus formation. Promotion and progression naturally are discussed, with particular reference to skin tumours, and foreignbody carcinogenesis occupies two chapters. In vitro techniques for the study of these processes, such as disruption of intercellular communication and cell transformation, do not have a current popularity, but they are not neglected here.

Because of the multiplicity of mechanisms which are likely to be involved, the study of nongenotoxic carcinogens lacks the unity of genetic toxicology and must attract researchers from a broad range of specialisations. Such a development is beneficial for the better understanding of neoplasia. This book serves as a focus for diverse activities and it is here that its importance rests. Its contents will not be entirely alien to anyone involved in carcinogenesis prediction or mechanism or carcinogen regulation, but its subject matter is a timely reminder of the complexity of these problems and the need for specialists from different backgrounds to listen to each other.

$$
\begin{array}{r}
\text { DOUGLAS MCGREGOR } \\
\text { Boehringer Ingelheim Pharmaceuticals Inc. } \\
\text { Ridgefield } \\
\text { USA }
\end{array}
$$

\section{Development Biology: A Comprehensive Synthesis Volume 4. Manipulation of Mammalian Develop- ment. Edited by R. B. L. GwatKIN. New York: Plenum Press. 1986. 388 pages. Subject index. \$59.50. ISBN 0306421666 .}

Dr R. Gwatkin did an excellent job. He invited chapters from many good scientists who had made great contributions to the problems under discussion. As a result, we have a book which deals not only with different ways of manipulating mammalian oocytes and embryos, but also with some theoretical problems likely to be of particular importance for those interested in new ideas and new results in the field of mammalian development.

Only a few years have passed since the publication of the 5-volume series on the development of mammals edited by $\mathrm{M}$. H. Johnson. The achievements made in this field since then have led to a breakthrough in our basic understanding, as well as to advances in animal breeding and in medicine. The book under review 